\title{
Construcción de un Calorímetro para la Determinación de Entalpías de Inmersión
}

\author{
Vanessa García(1) Juan Carlos Moreno-Piraján ${ }^{(1)}$ y Liliana Giraldo(2) \\ (1) Grupo de Investigación en sólidos porosos y calorimetría. Departamento de Química. \\ Facultad de Ciencias. Universidad de Los Andes, Carrera 1 No. 18 A 10, Bogotá-Colombia. \\ (e-mail jumoreno@uniandes.edu.co) \\ (2) Departamento de Química. Facultad de Ciencias. Universidad Nacional de Colombia, \\ Ciudad Universitaria, Carrera 30 No. 45 03, edificio 451, Bogotá-Colombia.
}

* autor a quien debe ser dirigida la correspondencia

\begin{abstract}
Resumen
Se presenta la construcción de un calorímetro de conducción de calor y se muestra en detalle cada uno de sus elementos constituyentes, con énfasis en el sistema que se usa para detectar el flujo de calor. El calorímetro fue eléctrica y químicamente calibrado y se determinó la entalpía de inmersión de carbones activados en soluciones acuosas de compuestos con capacidad de ionizarse, tales como fenol y nitrato de plomo. En el primer caso la entalpía de inmersión promedio resultó de $48.9 \pm$ $1.4 \mathrm{Jg}^{-1}$ para un carbón activado de área superficial $950 \mathrm{~m}^{2} \mathrm{~g}^{-1}$. En el segundo caso, las entalpías de inmersión variaron entre 5 y $65 \mathrm{Jg}^{-1}$ para distintas soluciones acuosas de $\mathrm{Pb}^{2+}$ y para un carbón activado con área superficial de $585 \mathrm{~m}^{2} \mathrm{~g}^{-1}$. Se concluye que el calorímetro construido tiene características de estabilidad de línea base, constante de calibración y sensibilidad, adecuadas para las determinaciones de entalpías de inmersión de sólidos porosos en líquidos.
\end{abstract}

Palabras clave: calorímetro, isotermas de adsorción, metales pesados, calor de inmersión

\section{Construction of a Calorimeter for the Determination of Immersion Enthalpies}

\begin{abstract}
The construction and use of a heat conduction calorimeter is presented, given specific details about every component, focusing in the system used to sensor the heat flow. The calorimeter was electrically and chemically calibrated and was used for determining of the immersion enthalpy of activated carbons in aqueous solutions of compounds with capacity of ionization, such as phenol and lead nitrate. In the first case, the immersion enthalpy of an activated carbon with superficial area of $950 \mathrm{~m}^{2} \mathrm{~g}^{-1}$ gave an average value of $48.9 \pm 1.4 \mathrm{Jg}^{-1}$. In the second case, $\mathrm{Pb}^{2+}$ aqueous solutions and an activated carbon with superficial area of $585 \mathrm{~m}^{2} \mathrm{~g}^{-1}$, the immersion enthalpies ranged between 5 and $65 \mathrm{Jg}^{-1}$. It is concluded that the constructed calorimeter has characteristic of base line stability, calibration constant and sensibility, appropriate to determine immersion enthalpies of porous solids in liquids.
\end{abstract}

Keywords: calorimeter, adsorption isotherm, heavy metals, immersion heats 


\section{INTRODUCCION}

La técnica calorimétrica es una de las más empleadas dentro de la termodinámica como una herramienta de utilidad para realizar la caracterización de los sistemas que generan o absorben energía térmica. Debido a la diversidad de sistemas y a la manera como se generan los efectos térmicos, se presentan diversidad de equipos calorimétricos, y es prácticamente imposible tener un único tipo de calorímetro que sea útil para realizar todas las determinaciones (Hohne et.al., 1996).

De acuerdo a la forma como se realiza la medida y al tipo de aislamiento que posea el calorímetro, estos pueden clasificarse como: calorímetros adiabáticos, isoperibólicos e isotérmicos, estos últimos contienen a los calorímetros de conducción de calor, que a su vez se clasifican como macro y micro calorímetros, donde normalmente los micro calorímetros hacen referencia al tamaño de la celda y a la sensibilidad en la determinación de la cantidad de calor del mismo (Hemminger et al., 1984: Wadso, 1994)

Los calorímetros isotérmicos, que corresponde al tipo de calorímetro que se describe en este trabajo, presentan un intercambio grande del calor que se produce en la celda con los alrededores; en este método los alrededores y la celda tienen la misma temperatura constante $\left(T_{A}=T_{C}=\right.$ constante). Así, el calorímetro posee una resistencia térmica $R_{T}$, entre celda y alrededores pequeña y además la capacidad calorífica de los alrededores es infinitamente grande. Si se tiene en cuenta dichos requisitos, en condiciones estrictamente isotérmicas $T_{A}$ y $T_{C}$ pueden permanecer constantes en el tiempo y en el espacio, pero entonces no se presenta flujo de calor. En los casos reales, se presenta un flujo de calor entre la celda y los alrededores, flujo que se detecta por medio de los sensores térmicos colocados entre ellos. El flujo es debido a la generalmente pequeña diferencia de temperatura existente entre $\mathrm{T}_{\mathrm{A}}$ y $\mathrm{T}_{\mathrm{C}}$ durante la ocurrencia del proceso observado; la magnitud de esta diferencia de temperatura depende de la cantidad de calor liberada por unidad de tiempo, de las conductividades térmicas y geometría de la celda y del tipo de aislamiento que posean los sensores térmicos (Wadso L 2001). A pesar de estas limitaciones, la designación isotérmica se usa comúnmente para calorímetros donde las temperaturas $T_{A}$ y $T_{C}$ pueden ser distintas entre sí, pero cada una de ellas considerada separadamente es constante durante todo el tiempo de ocurrencia del proceso que genera el flujo de calor (Hemminger et.al., 1984).

La conducción de calor en cuerpos sólidos permite medir cuantitativamente el intercambio de calor entre los alrededores y la celda de medida. Un registro en el tiempo de la diferencia de temperatura local suministra un medio para la medida del flujo de calor, si se conoce un factor de calibración específico. La resistencia térmica $R_{T}$, que conecta la celda con los alrededores es un parámetro interesante porque relaciona el flujo de calor, $\mathrm{dQ} / \mathrm{dt}$, con la diferencia de temperatura. La diferencia de temperatura en la resistencia térmica es:

$\Delta T=T_{C}-T_{A}$

y en el estado estacionario se presenta la relación:

$\frac{d Q}{d t}=\frac{\Delta T}{R_{T}}$

que al integrar se presenta como:

$Q=\frac{1}{R_{T}} \int \Delta T(t) d t$

Cuando $R_{T}$ es pequeña, el calor total fluye en un breve intervalo de tiempo, a través de $R_{T}$, hacia los alrededores. Así, el término $1 / R_{T}$ contribuye en gran forma al calor total.

Debido a la naturaleza compleja de la conducción de calor dentro de un instrumento real, es generalmente imposible calcular la resistencia $R_{T}$, que conecta de forma cuantitativa la diferencia de 
temperatura medida con el correspondiente flujo de calor; por esta razón la resistencia debe ser determinada por calibración (Wadso L, et.al 2001).

Puesto que la resistencia térmica también depende de la temperatura:

$Q=\int\left(\frac{\Delta T(t)}{R_{T}}\right) d t$

el valor recíproco de la resistencia térmica es el factor de calibración K(t):

$Q=\int\left(\frac{\Delta T(t)}{R_{T}}\right) d t$

En muchos casos el factor de calibración $\mathrm{K}(\mathrm{t})$ se puede registrar como constante en el rango de temperatura en el cual la reacción se lleva a cabo. El factor de calibración se calcula a partir de la relación entre el calor intercambiado y el área bajo la curva de medida. La calidad de un calorímetro depende esencialmente de la repetibilidad de este factor, cuando se varían otros parámetros del instrumento, tales como velocidad de calentamiento, intensidad, etc (Yff et.al 2004).

En nuestro grupo de trabajo, se han construido equipos calorimétricos de tipo isoperibólico e isotérmico (Girado et.al. 1994; 2002; Moreno et.al 1996; 2000; Granados et.al. 2004) con el propósito de medir las entalpías que producen varias interacciones entre las que se encuentran las que se generan entre sólidos y líquidos. Las entalpías de inmersión de sólidos porosos en líquidos, son características de un sistema y de unas condiciones experimentales específicas y sus valores suelen ser pequeños, del orden de Julios por gramo del sólido, de tal forma que se requiere de una instrumentación calorimétrica con la suficiente sensibilidad para evaluar los efectos térmicos que se generan cuando los componentes del sistema se ponen en contacto. (Wadso et al., 2001).

En este trabajo se muestra cada una de las partes y el ensamblaje de un microcalorímetro de conducción de calor, de tipo isotérmico, que usa termopilas como elementos sensores del flujo de calor celda-alrededores (Giraldo et. al, 2003), se presentan las constantes de calibración y las entalpías de reacción obtenidas con un sistema químico de referencia. Como resultados de interés se muestran las entalpías de inmersión de carbones activados en diferentes soluciones acuosas de compuestos iónicos como el fenol y nitrato de plomo y se observa la influencia de la temperatura como condición experimental en la entalpía de inmersión del carbón activado en soluciones acuosas de $\mathrm{Pb}^{2+}$ de diferente concentración.

\section{METODOLOGIA EXPERIMENTAL}

Buena parte de la metodología consiste en el diseño, elaboración y calibración de cada una de las partes que forman el microcalorímetro de conducción de calor. Con base en el concepto de conducción rápida del calor producido en la celda hacia los alrededores, tanto la celda como el depósito de calor se construyen en materiales metálicos y para evitar cambios en la respuesta del equipo por fluctuaciones térmicas de las condiciones externas la unidad calorímetrica, constituida por la celda, los sensores y el depósito de calor, se aislan mediante una cubierta plástica. A continuación se realiza la descripción detallada de las partes del microcalorímetro construido y la calibración que se realiza para que éste se utilice en las determinaciones de entalpías de inmersión.

\section{Celda Microcalorimétrica}

La celda calorimétrica se construye en acero inoxidable con una geometría cuadrada, cuyas dimensiones son 46,5 x 23,6 x 29,6 mm, que permite contener un volumen de aproximadamente 10 $\mathrm{mL}$ del líquido de inmersión. En la cara inferior de la celda, se coloca un cono terminado en punta con el objetivo que este sirva como elemento de contacto que ayuda al rompimiento de la ampolleta de vidrio, la cual contiene normalmente el sólido poroso, que en el caso de este trabajo es carbón activado. La Figura 1 muestra una fotografía de la celda y se hace referencia a sus dimensiones, 
como se observa en la parte superior la pieza tiene dimensiones superiores lo cual permite ubicarla y retirarla con facilidad del depósito de calor.

\section{Sensores Térmicos}

Para realizar el registro del calor que se genera dentro de la celda, se ensambla un circuito con cuatro termopilas unidas en serie, las cuales funcionan bajo el efecto Seebeck, y por tanto permiten realizar registros de potencial eléctrico de salida del calorímetro en función del tiempo (Giraldo et.al,2003). Las termopilas de materiales semiconductores empleadas en la calorimetría de conducción de calor son elementos de estado sólido, constituidas por una aleación cuaternaria de bismuto, teluro, selenio y antimonio, que proporciona al material un dopado y una orientación policristalina con propiedades termoeléctricas anisotrópicas (Moreno, 1997).

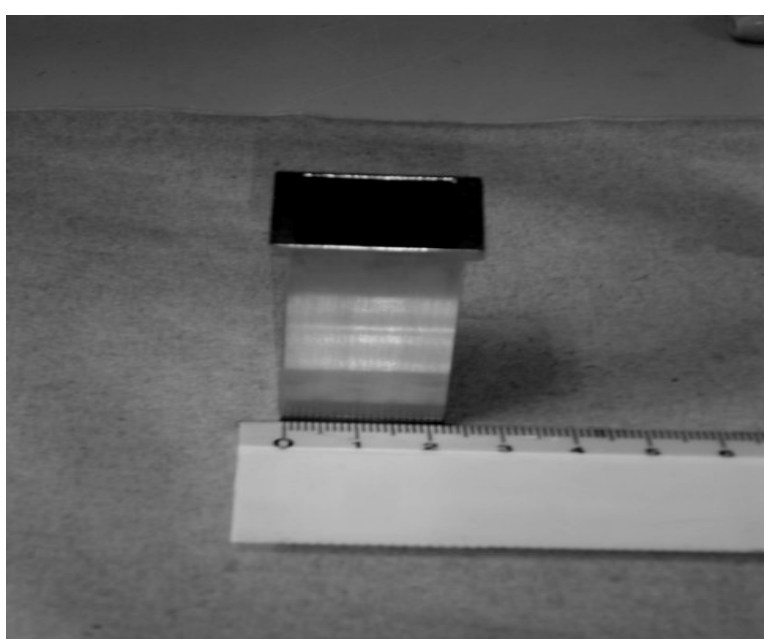

Fig. 1: Foto de la celda calorimétrica en acero inoxidable

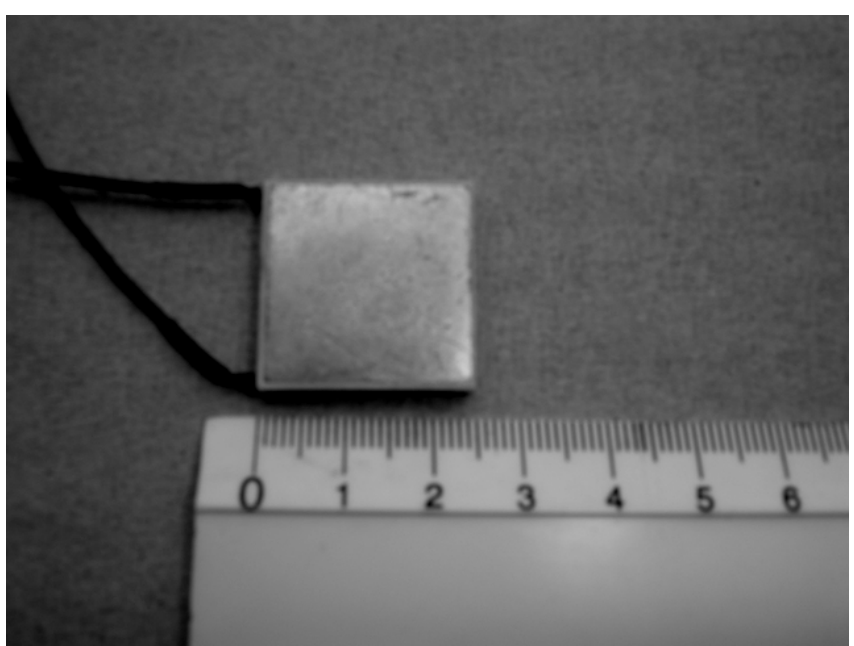

Fig. 2: Fotografía de una de las termopilas usadas como sensor del flujo de calor

Las termopilas con dimensiones de 25.0 x $25.0 \times 3,8 \mathrm{~mm}$ y una sensibilidad de $0.010 \mathrm{~W} \mathrm{~V}^{-1}$, se ubican rodeando la celda calorimétrica de tal manera que se deja únicamente un pequeño espacio para lograr en todo momento un buen contacto térmico para favorecer la conducción del calor. En la Figura 2, se muestra la fotografía de las termopilas que se usan en el calorímetro descrito; estos son termoelementos comerciales y que tienen presentaciones en las que varía la magnitud de su área de cubrimiento. La señal de potencial que se obtiene a la salida del calorímetro se registra a través de un multímetro de $61 / 2$ cifras Aligent modelo 34349 con una precisión de $0.1 \mu \mathrm{V}$, el cual se conecta a un computador por medio de un puerto RS-232, para la captura y posterior procesamiento de los datos.

\section{Deposito de Calor}

El depósito de calor está formado por una masa de aproximadamente $600 \mathrm{~g}$ de aluminio, que tiene como función lograr que la conducción de calor hacia los sensores se realice de una manera rápida (Wadso, 1988), gracias a la conductividad térmica del metal de $2.38 \mathrm{Jcm}^{-1} \mathrm{~s}^{-1} \mathrm{~K}^{-1}$. El depósito de calor es una barra maciza de $125 \mathrm{~mm}$ de diámetro por $100 \mathrm{~mm}$ de altura.

En el centro de la pieza se practican perforaciones con geometría similar a la de la celda y las termopilas en las que éstas se ubican; en buen contacto térmico, con ayuda de silicona térmica. En la Figura 3 se presenta una fotografía de la vista superior del depósito de calor, en la que se aprecian las perforaciones mencionadas anteriormente. 
Las conexiones eléctricas de las termopilas se realizan por la parte inferior del bloque metálico de tal forma que no se presente interferencia con éstas por la entrada y salida de la celda al depósito de calor cuando se lleva a cabo la experiencia calormétrica (Giraldo et.al., 2003).

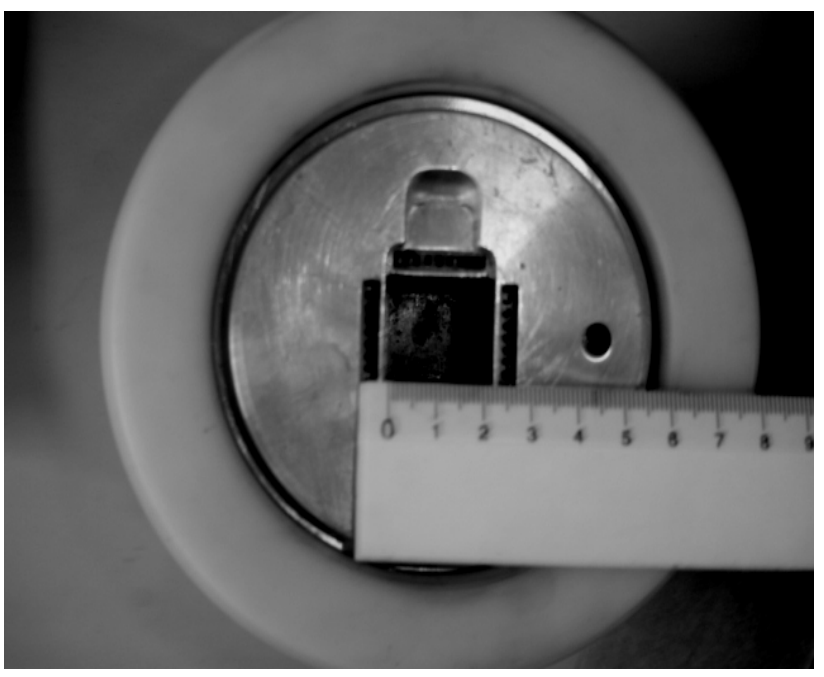

Fig. 3: Depósito de calor

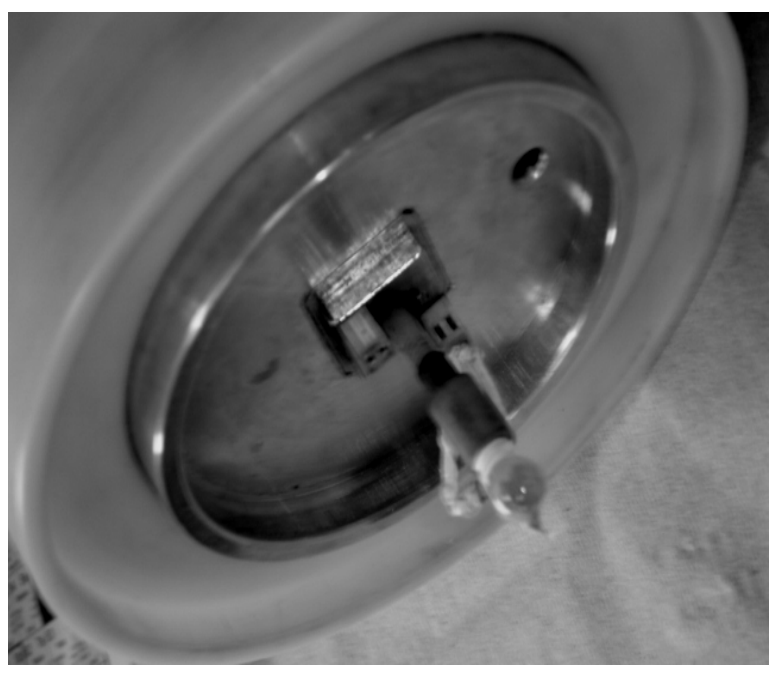

Fig. 4: Fotografía de la tapa del depósito de calor y ubicación de la ampolleta de vidrio.

El conjunto está provisto de una tapa, elaborada también en aluminio, que complementa el depósito de calor y en el que se instala un dispositivo que contiene la ampolleta de vidrio, en la que se coloca el material sólido, y que se opera desde el exterior para realizar el rompimiento de ésta una vez se alcanza estabilidad térmica y de la señal de salida del equipo.

En la Figura 4 se muestra la tapa del depósito de calor y la forma como se acondiciona la ampolleta de vidrio. La tapa también contiene una resistencia eléctrica de 100 ohmios y $0.25 \mathrm{~W}$, que se usa para la calibración de la unidad calorimétrica, para la obtención de los parámetros instrumentales como constante del calorímetro, K, y sensibilidad, S (Russell et.al., 06).

\section{Escudo Aislante térmico}

Para lograr una buena estabilización de la señal de potencial de salida del equipo se aisla el depósito de calor con un material plástico, nylon, de tal forma que se obtenga un conjunto compacto con un espesor de la capa plástica de $25 \mathrm{~mm}$, con respecto al diámetro de la pieza metálica. Las partes superior e inferior también se cubre con el mismo propósito. La Figura 5 , muestra el conjunto y la forma como se realizan las conexiones eléctricas de la resistencia de calibración y la salida del circuito de termopilas.

Las experiencias calorimétricas se llevan a cabo a temperatura constante, para condiciones cercanas al ambiente; por esto el conjunto descrito anteriormente se coloca en un termostato de aire, elaborado en acrílico, con un volumen aproximado de $4 \mathrm{~L}$.

\section{Determinación de la constante del equipo}

La constante del calorímetro se puede obtener mediante dos métodos: uno consiste en disipar una cantidad de trabajo eléctrico conocido dentro de la celda y registrar la señal de potencial de salida del calorímetro en función del tiempo; la cantidad de energía que se suministra a la celda genera un pico en la gráfica, de este modo la constante, $\mathrm{K}$, del calorímetro se calcula como la relación entre el trabajo eléctrico conocido y el área bajo la curva del pico (Steckler et.al, 1986; Giraldo et.al, 2004). Se registran curvas de potencial en función del tiempo para cantidades crecientes de trabajo eléctrico en un rango que varía desde 5 hasta $50 \mathrm{~J}$. 
La otra forma de determinar la constante, es disipar una potencia eléctrica estacionaria en la celda, lo cual generara un potencial de estado estacionario a la salida del equipo, la constante, $\mathrm{K}$, es la relación entre estas dos magnitudes generadas en estado estacionario. Se registran curvas de potencial de salida del equipo en función del tiempo, hasta alcanzar las condiciones estacionarias para valores de potencia eléctrica que varían entre 5 a $18 \mathrm{~mW}$.

\section{Caracterización química del equipo}

Como sistema químico de referencia se emplea la reacción de neutralización de $\mathrm{HCl}$ con $\mathrm{NaOH}$, reactivos Merck a partir de los cuales se preparan las soluciones (Kegeles, 1941; Wadso et.al 2001). Se determina la curva de potencial de salida del equipo en función del tiempo en la que la reacción de neutralización se realiza mediante el rompimiento de la ampolleta de vidrio y finalmente se compara con un trabajo eléctrico.

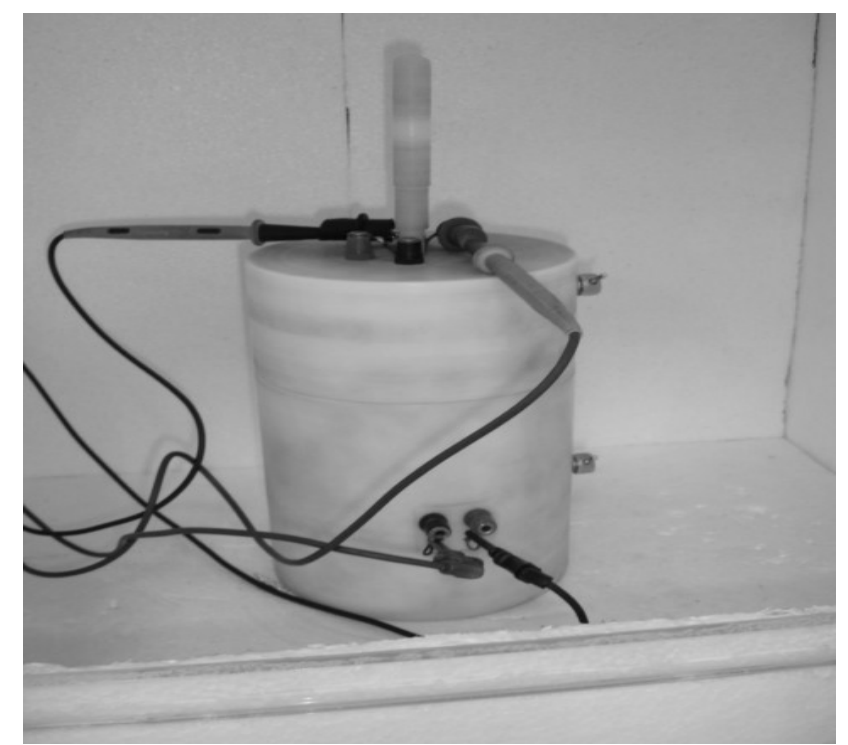

Fig. 5: Conjunto calorimétrico

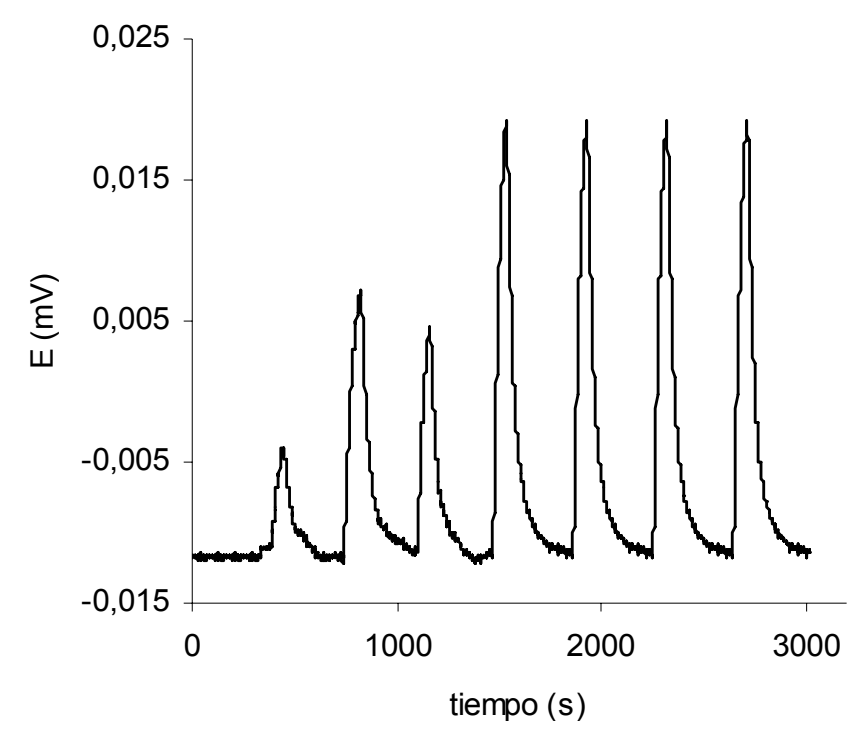

Fig. 6: Potencial de salida del equipo en función del tiempo.

\section{Determinación de la entalpía de inmersión de carbones activados en soluciones acuosas}

Una vez que se conocen las constantes instrumentales del calorímetro construido y se verifica, mediante la determinación de la entalpía de reacción de un sistema estándar, su correcto funcionamiento; éste se utiliza en la determinación de las entalpías de inmersión de carbones activados, que son materiales porosos, en soluciones de solutos iónicos, en un caso fenol y en el otro $\mathrm{Pb}^{2+}$. Los resultados obtenidos se presentan como uno de los campos de aplicación para el calorímetro de conducción elaborado; inicialmente se presentan los carbones activados utilizados en cada caso y la preparación de las soluciones y por último se describe la técnica calorimétrica.

Para la determinación de la entalpía de inmersión del carbón activado en soluciones de $\mathrm{Pb}^{2+}$, se usa un carbón activado obtenido a partir de cascarilla de café por activación química del precursor, mediante impregnación con una solución de $\mathrm{KOH}$ de $35 \%$ y posterior carbonización a una temperatura de $273 \mathrm{~K}$ durante media hora (Baquero et.al, 2003). El área superficial del sólido es de $585 \mathrm{~m}^{2} \mathrm{~g}^{-1}$. Las soluciones que se emplean en las determinaciones calorimétricas, se preparan a partir de una solución inicial de $1000 \mathrm{mgL}^{-1}$ de $\mathrm{Pb}\left(\mathrm{NO}_{3}\right)_{2}$, reactivo analítico Carlo Erba $99,5 \%$ de pureza, y se obtienen por dilución con una solución de $\mathrm{HNO}_{3}$ al $1 \%$ soluciones del ión $\mathrm{Pb}^{2+}$ en un rango de 20 a $120 \mathrm{mgL}^{-1}$. La concentración del ión $\mathrm{Pb}^{2+}$ en solución acuosa se determina por espectrofotometría de absorción atómica en un equipo Perkin Elmer Analyst 300 provisto con el software AA Win Lab Analyst, previa calibración. 
En el caso de la determinación de la entalpía de inmersión de un carbón activado en solución de 100 $\mathrm{mgL}^{-1}$ de fenol, Merck reactivo analítico, el sólido carbonoso que se estudia es un carbón activado comercial, producido por Polytrade a partir de cáscara de coco, con un área superficial de $950 \mathrm{~m}^{2} \mathrm{~g}^{-1}$. Se pesa aproximadamente $250 \mathrm{mg}$ del carbón activado, se colocan en la ampolleta de vidrio y se desgasifica a $573 \mathrm{~K}$ durante 48 horas. Luego de lo cual la ampolleta se sella y se coloca dentro de la celda calorimétrica, en la que se añaden $10 \mathrm{~mL}$ del líquido calorimétrico en el que se realiza la inmersión. (Moreno, 2004; López-Ramón et.al,1999;).

\section{RESULTADOS Y DISCUSIÓN}

Como resultado de la calibración eléctrica se obtiene la constante, $\mathrm{K}$, del calorímetro que está directamente relacionada con la sensibilidad del mismo; en la Tabla 1 se muestran los resultados para dicha constante, cuando se emplea el método de suministro de trabajo eléctrico

Tabla 1: Determinación de la constante del calorímetro

\begin{tabular}{|c|c|c|}
\hline Trabajo eléctrico $(\mathrm{J})$ & Constante $\mathrm{K}\left(\mathrm{W} \mathrm{V}^{-1}\right)$ & Desviación estándar $\left(\mathrm{W} \mathrm{V}^{-1}\right)$ \\
\hline 5 & 15.24 & 0.28 \\
\hline 10 & 13.15 & 0.43 \\
\hline 30 & 13.29 & 0.37 \\
\hline 50 & 13.18 & 0.32 \\
\hline
\end{tabular}

La variación en el pulso de trabajo eléctrico, entre 5 y $50 \mathrm{~J}$, que se suministra a la celda está relacionado con la magnitud de calor que se produce cuando se ponen en contacto un sólido poroso y un líquido (Silvestre et.al, 2001); se observa que para el valor más bajo de trabajo eléctrico se presenta un valor mayor para $\mathrm{K}$, de $15.24 \mathrm{~W} \mathrm{~V}^{-1}$, que para las adiciones de energía mayor cuyos valores se encuentran alrededor de $13 \mathrm{~W} \mathrm{~V}^{-1}$. De acuerdo a estos resultados, los valores para la sensibilidad del calorímetro se presentan entre 0.066 y $0.076 \mathrm{~V} \mathrm{~W}^{-1}$, que están de acuerdo con datos de la literatura (Steckler et.al,1986; ).

En la Figura 6, se muestra una gráfica de potencial de la salida del equipo en función del tiempo, en la que se realizan varias adiciones de diferentes cantidades de trabajo eléctrico, Welec. Se observa en la Figura 6 que los picos presentan áreas diferentes para diferentes cantidades de trabajo eléctrico y que para la adición de una misma cantidad de trabajo eléctrico, que se presenta para los últimos cuatro picos, el valor del área se hace reproducible; la respuesta del sistema es apreciable y se cuenta con una estabilidad de la línea base alrededor de $5 \mu \mathrm{V}$.

La otra forma como se determina el valor de la constante $\mathrm{K}$ del equipo, es por el método de estado estacionario, para este caso se disipan en la celda calorimétrica cantidades de potencia eléctrica entre aproximadamente 5 a $18 \mathrm{~mW}$. En la Figura 7 se muestran algunas curvas obtenidas, de potencial de salida del equipo en función del tiempo, para tres potencias de calentamiento distintas y se aprecia como se alcanza un potencial de equilibrio que depende de las condiciones de calentamiento y aumentan el potencial estacionario desde 0.388 hasta $0.856 \mathrm{mV}$. La constante $\mathrm{K}$, se puede obtener como de la pendiente de una gráfica de potencia estacionaria de calentamiento en función del potencial de salida del equipo estacionario. En la Figura 8 se muestra este tipo de representación. Se observa en la Figura 8 un comportamiento lineal, con un valor para la pendiente de $13.15 \mathrm{~W} \mathrm{~V}^{-1}$ y un coeficiente de correlación de 0.9995 ; se muestra que en el calorímetro se presentan variaciones de potencial apreciables y con valores superiores a los de estabilidad de línea base para cantidades pequeñas de potencias de calentamiento. En la Tabla 2 se presenta un resumen de los resultados obtenidos para la calibración del equipo en las condiciones que se han descrito anteriormente. Un resultado interesante es el de la estabilidad en la línea base ya que tanto en condiciones de temperatura constante como en calentamiento el valor es el mismo y los niveles de ruido se mantienen controlados. 


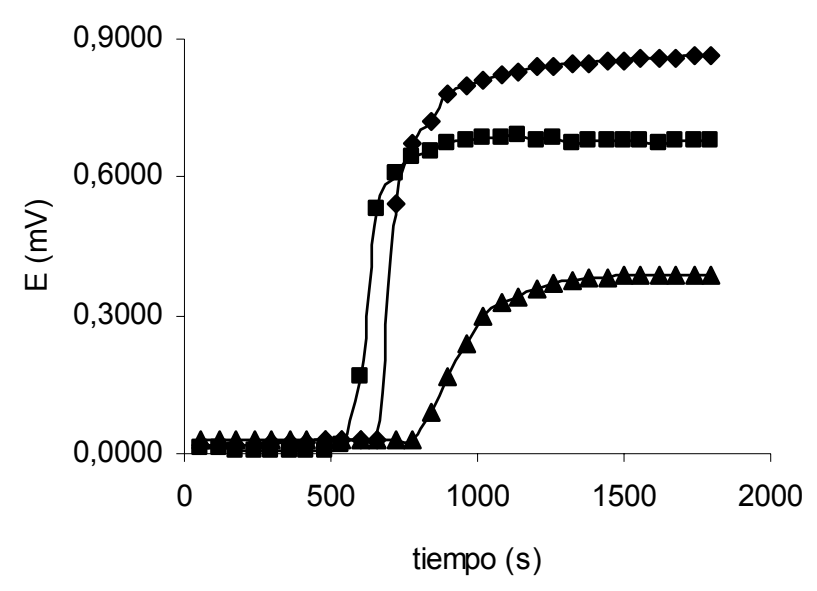

Fig. 7: Curvas de potencial de salida del equipo. Potencias de calentamiento $\mathbf{\Delta} 5.11$ $\mathrm{mW}=8.95 \mathrm{~mW} \bullet 11.26 \mathrm{~mW}$

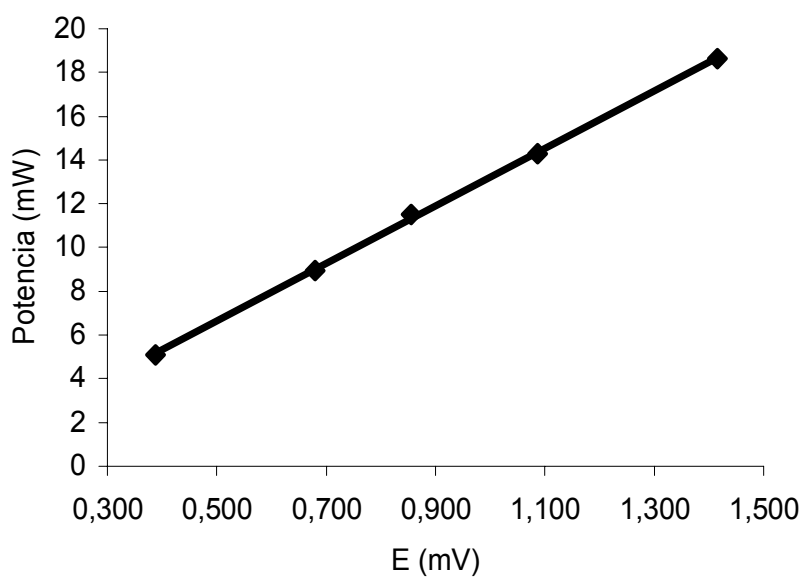

Fig. 8: Potencia estacionaria de calentamiento en función del potencial de salida del equipo

Tabla 2: Resultados de la calibración del calorímetro

\begin{tabular}{|l|l|}
\hline Estabilidad de línea base & $5 \mu \mathrm{V}$ \\
\hline $\begin{array}{l}\text { Estabilidad de línea base en condiciones } \\
\text { de calentamiento }\end{array}$ & $5 \mu \mathrm{V}$ \\
\hline Constante $\mathrm{K}$ & 13.15 a $15.24 \mathrm{~W} \mathrm{~V}^{-1}$ \\
\hline Desviación estándar & 0.43 a $0.28 \mathrm{WV}^{-1}$ \\
\hline Sensibilidad & 0.066 a $0.076 \mathrm{~V} \mathrm{~W}^{-1}$ \\
\hline
\end{tabular}

Uno de los sistemas que se ha utilizado ampliamente como estándar de comparación en calorimetría es la entalpía de neutralización de $\mathrm{HCl}$ con $\mathrm{NaOH}$, debido a su alto valor y a las características de la reacción. Como una forma de llevar a cabo la calibración y comprobar el correcto funcionamiento del equipo, se realizan 30 determinaciones de la entalpía de neutralización del sistema $\mathrm{HCl}-\mathrm{NaOH}$ y se obtiene un valor de $-56.57 \pm 0.01 \mathrm{kJmol}^{-1}$, que está en buen acuerdo con la literatura (Kegeles, 1941; Giraldo et.al, 2003) y que presenta una buena reproducibilidad. Algunos de los resultados obtenidos se presentan en la Tabla 3, en la que registra la cantidad en moles de $\mathrm{HCl}$ que reacciona, el calor que se genera en la reacción, $\mathrm{Q}$ en $\mathrm{mJ}$ y la entalpía de neutralización, $\Delta \mathrm{H}$ en $\mathrm{Jmol}^{-1}$.

Cuando un sólido se sumerge en un líquido con el cual no reacciona, se genera cierta cantidad de calor; esta entalpía de inmersión se relaciona con la formación de una capa de moléculas adsorbidas en la superficie del sólido. Las entalpías de inmersión de un sólido en diferentes líquidos normalmente son diferentes; ya que ésta no sólo está relacionada con al área superficial aprovechable por el líquido, sino por las interacciones específicas entre la superficie del sólido y el líquido de inmersión (Ruoquerol et.al, 1999). Por medio de la calorimetría de inmersión es posible establecer relaciones entre el calor generado en el contacto sólido-líquido y las áreas superficiales de sólidos porosos, por ejemplo al sumergir el sólido en un solvente, generalmente de tipo no polar (Stoeckli et.al., 1997). Así mismo, cuando se desea tener información sobre los efectos térmicos que se presentan entre el sólido y el líquido, se pueden utilizar soluciones de ácidos, bases y otros solutos para conocer la interacción energética con grupos funcionales que se encuentren en la superficie del sólido (López-Ramón et.al, 1999). Teniendo en cuenta lo anterior, se muestran resultados obtenidos en la determinación de la entalpía de inmersión de carbones activados en soluciones acuosas; el calor que se genera es la sumatoria de las interacciones entre el sólido y el líquido, que en este caso está representado por el solvente y el soluto. Se puede entonces relacionar dicho calor por gramo del sólido poroso, y obtener la entalpía de inmersión en las condiciones experimentales específicas, que serán características del sistema. 
Tabla 3: Determinación de la entalpía de neutralización sistema $\mathrm{HCl}-\mathrm{NaOH}$

\begin{tabular}{|l|l|l|}
\hline Moles de $\mathrm{HCl}$ & - Calor, $\mathrm{Q}(\mathrm{mJ})$ & $-\Delta \mathrm{H}\left(\mathrm{Jmol}^{-1}\right)$ \\
\hline 0.4518 & 25.6 & 56.66 \\
\hline 0.4623 & 26.2 & 56.67 \\
\hline 0.4767 & 27.0 & 56.63 \\
\hline 0.4555 & 25.8 & 56.64 \\
\hline 0.4628 & 26.2 & 56.61 \\
\hline 0.4837 & 27.4 & 56.64 \\
\hline 0.4520 & 25.6 & 56.63 \\
\hline 0.4626 & 26.2 & 56.64 \\
\hline 0.4130 & 23.4 & 56.66 \\
\hline
\end{tabular}

La Figura 9 muestra un potenciograma típico obtenido en la inmersión de una muestra del carbón activado comercial Polytrade en una solución acuosa de fenol de $100 \mathrm{mg} \mathrm{mL}^{-1}$ a $298 \mathrm{~K}$. Como se puede observar el primer pico corresponde al efecto térmico de la inmersión y el segundo a la calibración eléctrica, se destaca la definición de la línea base y la nitidez en el desarrollo de los efectos.

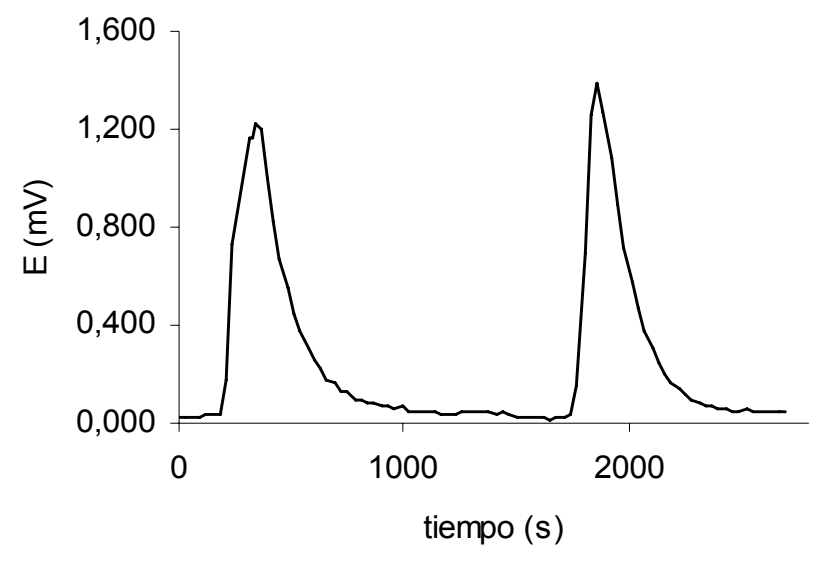

Fig. 9: Curva de potencial para la inmersión de carbón activado comercial en solución de fenol.

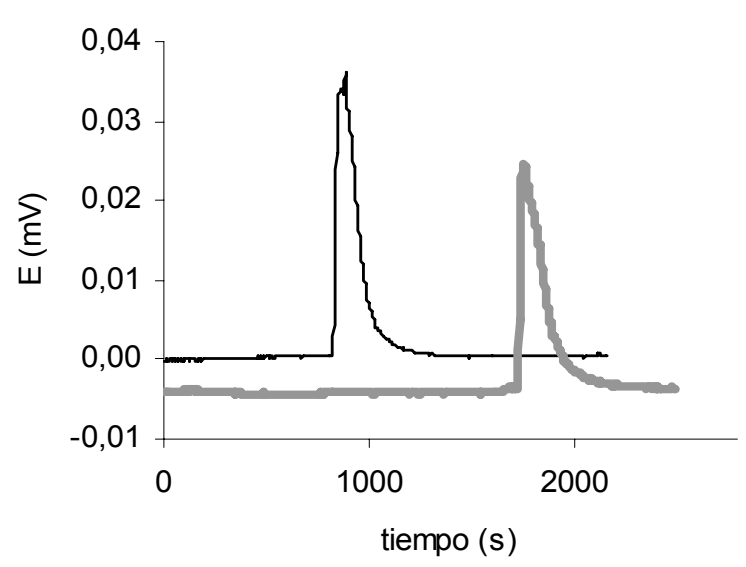

Fig. 10: Termogramas de inmersión de carbón activado obtenido de cascarilla de café en soluciones acuosas de $\mathrm{Pb}^{2+}$. $298 \mathrm{~K}$ $318 \mathrm{~K}$

El valor obtenido para la entalpía de inmersión del carbón activado Polytrade en solución de 100 $\mathrm{mgL}-1$ de fenol es de $48.9 \pm 1.4 \mathrm{Jg}-1$, resultado promedio de tres determinaciones. Este valor es comparable con el que se presenta en la literatura para la inmersión de carbones activados en agua y soluciones básicas (Steckler et.al,1986), aunque se debe tener en cuenta que las interacciones entre el fenol, que actúa como soluto, depende del contenido de grupos superficiales de oxígeno (Dabrowski et.al., 2005)

Finalmente, se muestran los resultados obtenidos para la determinación de la entalpía de inmersión de carbón activado obtenido a partir de cascarilla de café, en soluciones de diferente concentración de $\mathrm{Pb}^{2+}$ y se observa la influencia de la temperatura en la entalpía de inmersión, al determinar ésta a 298 y 318 K. En la Figura 10 se muestra termogramas obtenidos para la inmersión del carbón activado a las dos temperaturas propuestas, en una solución de $80 \mathrm{mgL}^{-1}$. Se observa en la gráfica que el efecto térmico que se genera es mayor a temperatura de $298 \mathrm{~K}$, que para la temperatura superior de $318 \mathrm{~K}$ y esto está de acuerdo con los resultados obtenidos para la capacidad de adsorción a tales temperaturas. 
En la Figura 11, se muestra que la entalpía de inmersión en función de la concentración es mayor a la $298 \mathrm{~K}$ que a $318 \mathrm{~K}$, aunque para las concentraciones de 20 y $40 \mathrm{mgL}^{-1}$, los valores son similares y posteriormente la diferencia aumenta hasta alcanzar un valor constante. En la Figura 11 se presenta la relación entre la entalpía de inmersión del carbón activado en soluciones del ión metálico en función de la concentración de la solución; estos ensayos se realizan igualmente para las temperaturas que se han propuesto.

Se observa, para cada una de las temperaturas, que las entalpías de inmersión aumentan para concentraciones intermedias y entre 100 y $120 \mathrm{mgL}^{-1}$ los valores de ésta tienden a un valor constante, que es un comportamiento similar al que se obtiene para la isoterma de adsorción; se puede entonces, hacer un seguimiento del proceso de adsorción midiendo las entalpías de inmersión, ya que éstas reflejan la intensidad de la interacción, que como se dijo son de carácter exotérmico y para determinadas concentraciones del ión, la entalpía de inmersión se ubica en la región de equilibrio para un sistema a una condiciones experimentales específicas.

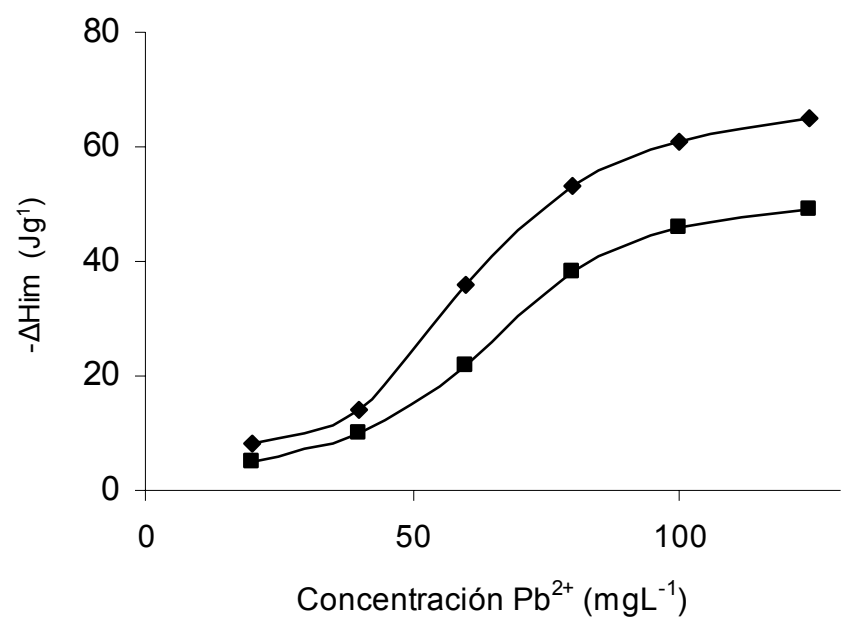

Fig. 11: Influencia de la temperatura en la curva de entalpía de inmersión en función de la concentración de $\mathrm{Pb}^{2+}$. $298 \mathrm{~K} ;$ a $318 \mathrm{~K}$

\section{CONCLUSIONES}

Se construye un microcalorímetro de conducción de calor, que usa termopilas como sensores del flujo de calor. La celda calorimétrica y el depósito de calor son metálicos para asegurar la rápida conducción de calor celda-alrededores. Se obtiene adecuadas características instrumentales, como estabilidad de línea base de $5 \mu \mathrm{V}$, constante de calibración de entre $13.15 \pm 0.43$ y $15.24 \pm 0.28$ W. $\mathrm{V}^{-1}$ y sensibilidad de $0.066 \mathrm{VW}^{-1}$, adecuadas para las determinaciones de entalpías de inmersión de sólidos porosos en líquidos. La calibración química se realiza por la determinación de la entalpía de neutralización de $\mathrm{HCl}$ con $\mathrm{NaOH}$, para la que se obtiene un valor de $-56.64 \pm 0.01 \mathrm{kJmol}^{-1}$, que está en concordancia con los resultados de literatura. Se miden las entalpías de inmersión de un carbón activado obtenido de cáscara de coco, en una solución acuosa de fenol, para la que se obtiene en valor de $48.9 \pm 1.4 \mathrm{Jg}^{-1}$, que muestra la capacidad del calorímetro en la determinación de cantidades de calor pequeñas. Se determinan entalpías de inmersión de carbón activado obtenido de cascarilla de café, en soluciones acuosas de $\mathrm{Pb}^{2+}$ en función de la concentración de y de la temperatura 298 y $318 \mathrm{~K}$, con valores que se encuentran entre 5 y $65 \mathrm{Jg}^{-1}$, y que presenta entalpías de inmersión mayores para la temperatura menor.

\section{AGRADECIMIENTOS}

Los autores agradecen al Convenio Marco entre la Universidad de los Andes y la Universidad Nacional de Colombia. Un agradecimiento especial al Fondo de Investigaciones de la Facultad de Ciencias de la Universidad de Los Andes por la financiación parcial de ésta investigación. 


\section{REFERENCIAS}

Baquero, M.C., L. Giraldo y J.C. Moreno, Determination of the immersion enthalpy of activated carbons in polar and apolar liquids. Electronic Journal of Environmental, Agricultural and Food Chemistry, 4(4), 1-11, 2005.http://ejeafche.uvigo.es/4(4)2005/004442005F.htm

Dabrowski, A., P. Podkoscienlny, Z. Hubicki y M. Barczak, Adsorption of phenolic compounds by activated carbon, Chemosphere 58(8), 1049 (2005)

Giraldo, L., J.C. Moreno y A. Gómez, Construcción de un calorímetro isoperibólico de inmersión de precisión, Rev. Col. Quim. 23(1), 1-14 (1994)

Giraldo, L., J.C. Moreno y J.I. Huertas, Heats Conduction Micro-Calorimeter With Metallic Reaction Cells, Instrumentation Science \& Technology. 30 (2), 177-186 (2002)

Giraldo, L. y J.C. Moreno., Determinación de la entalpía de inmersión de carbón activado en soluciones acuosas de fenol y su relación con la capacidad de adsorción. Revista Colombiana de Química, 32(1), 45-54 (2003)

Giraldo, L. y J.C. Moreno., Determinación de la entalpía de inmersión y capacidad de adsorción de un carbón activado en soluciones acuosas de plomo. Rev. Col. Quim. 33(2), 87-97 (2004)

Granados, C., N. Rojas, L. Giraldo y J.C. Moreno, Preliminary exploration of the behavior of conduction calorimetric cells in the determination of the caloric value of mnicipal solid waste, Instrumentation Science \& Technology, 32(2), 139-152 (2004)

Hemminger W., y G. Hohne., Calorimetry fundamentals and practice. $2^{\mathrm{a}}$ Edition, 121-123. Verlag Chemie, Florida (1984)

Hohne, G.W.H., W. Hemminger y H.J. Flammersheim, Differencial Scanning Calorimetry. An Introduction for practitioners. $1^{\text {a }}$ Edition, 193-194, Springer; Berlin (1996)

Kegeles G., The heat of neutralization of sodium hydroxide with hydrochloric acid. J. Am. Chem. Soc., 62,3230 (1941)

Lopez-Ramón. M., F. Stoeckli., C.C. Moreno., M. F. Carrasco., On the Characterization of Acidic and Basic Surface Sites on Carbons by Various Techniques. Carbon, 37, 1215-1221 (1999)

Moreno, C.C., Adsorption of organic molecules from aqueous solutions on carbon materials, Carbon, 42, 83-94 (2004)

Moreno, J.C., L. Giraldo y A. Gómez, A batch type heat conduction microcalorimeter for immersion heat determinations: design and calibration, Thermochim. Acta, 290, 1-12 (1996)

Moreno, J.C., Diseño, construcción y calibración de un microcalorímetro de conducción de calor y su aplicación al estudio de sólidos porosos. Tesis de Doctorado. Universidad Nacional de Colombia. Bogotá. (1997)

Moreno, J.C. y L. Giraldo, Determination of the immersion enthalpy of activated carbon by microcalorimetry of the heat conduction, Instrumentation Science \& Technology, 28(2), 171- 178 (2000)

Rouquerol, F., J. Rouquerol y K.S.W. Sing, Adsorption by powders and porous solids. Principles, methodology and applications, Academic Press, 167-170, San Diego (1999)

Russell, D.J. y L.D. Hansen, Calorimeters for biotechnology, Thermochim. Acta, 445, 151-159 (2006) 
Silvestre-Albero, J., C. Gómez, A. Sepúlveda-Escribano y F. Rodríguez-Reinoso, Characterization of microporous solids by inmersion calorimetry. Colloids and surfaces A, 187, 151-165 (2001)

Steckler, D.K., Golberg, R.N., Tewari, Y.B. y Buckley T.J., High precision microcalorimetry: apparatus, procesures, and biochemical applications, J. Res. Natl. Bur. Stand. U.S.A., 91, 113-121 (1986)

Stoeckli, H.F. y T.A. Centeno, On the characterization of microporous carbons by immersion calorimetry alone, Carbon, 35, 1097-1100 (1997)

Wadso, I., Chapter 6, Thermochemistry of living cell systems. in Biochemical Thermodynamics. Jones M.N. (Ed.). Elsevier. 2a ed. Amsterdam. (1988)

Wadso, I., Microcalorimetry of aqueous and biological systems, in Solution Calorimetry, K.N. Marsh and P.A.G. O'Hare (Editors), IUPAC. Commission on Thermodynamics, Blackwell Scientific Publications, 267-299, Oxford (1994)

Wadso, I. y R.N. Goldberg, Standards in isothermal microcalorimetry. Pure Appl. Chem., 73(10), 1625-1639 (2001)

Wadso, L., A.L. Smith, H. Shirazi, S.R. Mulligan y T. Hofelich, The isothermal heat conduction calorimeter: a versatile instrument for studying processes in physics, chemistry and biology., J. Chem. Educ. 78 (8), 1080-1086 (2001) 